\title{
Aspectos da morfologia radiográfica do esqueleto, tórax e abdome do quati (Nasua nasua Linnaeus, 1766) ${ }^{1}$
}

\author{
Gisele S. Martins², Erika R. Lopes ${ }^{3}$, Isis I.G. Taques², Cristiane Y. Correia², Yara S. Meireles², \\ Nívea C.M.R. Turbino ${ }^{2}$, Luciana D. Guimarães ${ }^{2}$ e Pedro Brandini Néspoli ${ }^{2 *}$
}

\begin{abstract}
Martins G.S., Lopes E.R., Taques I.I.G., Correia C.Y., Meireles Y.S., Turbino N.C.M.R., Guimarães L.D. \& Néspoli P.B. 2013. [Radiographic morphology of the skeleton, thorax and abdomen of coati (Nasua nasua Linnaeus, 1766).] Aspectos da morfologia radiográfica do esqueleto, tórax e abdome do quati (Nasua nasua Linnaeus, 1766). Pesquisa Veterinária Brasileira 33(9):1137-1143. Setor de diagnóstico por Imagem, Hospital Veterinário, Universidade Federal de Mato Grosso, Av. Fernando Corrêa da Costa 2367, Bairro Boa Esperança, Cuiabá, MT 78-60-900, Brazil.E-mail: nespoli@ufmt.br

Thirteen adult coatis (Nasua nasua) from Zoological Park of the Federal University of Mato Grosso were chemically restrained and subjected to several radiographic procedures. Radiographs of the fore limbs, hind limbs, thorax, abdomen, neck and skull were performed. The images obtained were compared to anatomical specimens and to radiographs of dogs. Descriptions of the radiographic morphology of internal organs and skeletal structures were performed and images of the main radiographs of the species were made available. The main differences between coatis and canines members radiographic morphology were limited to the hands and feet. Coatis had 5 well developed digits with the metacarpals and metatarsals slightly shorter than the corresponding digits. This species have 7 cervical vertebrae, 15 thoracic, 5 lumbar vertebrae, and 3 sacral (fused). The frontal sinuses were larger, with cranial extension between the maxillary and nasal bones, numerous and very evident septa. The observed dentition was I 3/3, C1 / 1, P4 / 4 M2 / $2=40$ and the main thoracic and ventral organs showed similar anatomy and radiographic features to those described for canines.
\end{abstract}

INDEX TERMS: Coati, Nasua nasua, radiology, anatomy, morfology.

RESUMO.- Treze quatis (Nasua nasua) oriundos do Parque Zoológico da Universidade Federal do Mato Grosso foram contidos quimicamente e submetidos a diversos procedimentos radiográficos. Foram realizadas radiografias dos membros torácicos, membros pélvicos, tórax, abdome, pescoço e crânio. As imagens obtidas foram comparadas com peça anatômica e com imagens radiográficas de caninos. Foram realizadas descrições da morfologia radiográfica de vísceras e de estruturas esqueléticas e disponibilizadas imagens das principais projeções radiográficas da espécie.

\footnotetext{
${ }^{1}$ Recebido em 27 de fevereiro de 2013.

Aceito para publicação em 19 de julho de 2013.

${ }^{2}$ Av. Felinto Müller 2356, Bairro Nova Várzea Grande, Várzea Grande, Cuiabá, MT 78135 000, Brasil E-mail: salengue@gmail.com

${ }^{3}$ Setor de Diagnóstico por Imagem, Hospital Veterinário, Universidade Federal de Mato Grosso (UFMT), Av. Fernando Corrêa da Costa 2367, Cuiabá, MT 78060-900. *Autor para correspondência: nespoli@ufmt.br

${ }^{4}$ Centro Cirúrgico, Hospital Veterinário, UFMT, Cuiabá, MT. E-mail: nmrochaturbino@hotmail.com
}

As principais diferenças entre a morfologia radiográfica dos membros de quatis e de caninos ficaram limitadas às mãos e aos pés. Os quatis apresentaram 5 dígitos bem desenvolvidos com os metacarpos e os metatarsos levemente mais curtos do que os dígitos correspondentes. Essa espécie apresentou 7 vértebras cervicais, 15 torácicas, 5 vértebras lombares e 3 sacrais (fusionadas). Os seios frontais mostraram-se mais amplos, com extensão cranial entre o osso maxilar e o nasal, e numerosos septos bem evidentes. A dentição observada foi I 3/3, C1/1, P4/4, M2/2 = 40 e as principais vísceras torácicas e abdominais apresentaram aspectos anatômico e radiográfico similares às descritas para caninos.

TERMOS DE INDEXAÇÃO: Quati, Nasua nasua, radiologia, anatomia, morfologia.

\section{INTRODUÇÃO}

A radiologia é bastante eficaz na identificação de alterações ósseas, do trato respiratório, digestivo e urinário tanto de 
animais domésticos quanto de animais silvestres e amplia consideravelmente as chances de sucesso do diagnóstico clinico (Fonseca Pinto 2007).

Entretanto, a interpretação adequada das imagens e a melhor prática radiológica precisam combinar experiência, conhecimento de anatomia radiográfica (Schebitz \& Wilkens 2000, Kealy \& McAllister 2005), compreensão dos processos fisiológicos e patológicos e a consideração do quadro clínico (Kealy \& McAllister 2005).

Atualmente a falta de conhecimento da anatomia radiográfica de animais silvestres ainda é um dos elementos que dificultam a interpretação dos exames desses animais. Mesmo quando se procura agrupar os animais por similaridade dentro das classes (aves, répteis e mamíferos), persiste ainda uma incalculável variação anatômica entre os membros de cada grupo (Fonseca Pinto 2007). Assim, mesmo com o grande avanço das últimas três décadas, a Medicina Veterinária de Animais Silvestres ainda detém uma bibliografia restrita (Cubas et al. 2007) tanto no que se refere à pesquisa básica quanto à aplicada.

Em relação ao quati (Ordem Carnivora, família Procyonidae, subfamília Procioninae) não é diferente. Apesar de ser um animal relativamente bem estudado, com trabalhos conduzidos nas áreas de morfologia (Franciolli et al. 2007, Gregores et al. 2010, Santos et al. 2010), ecologia (Beisiegel 2001) e diagnóstico de enfermidades, não existem estudos amplos sobre a sua anatomia radiográfica. Dessa forma, o diagnóstico radiográfico de doenças que afetam esses animais, sobretudo em condições de cativeiro, como as lesões dentárias (Freitas et al. 2008), dioctofimose (Kommers et al. 1999, Milanelo et al. 2009), tuberculose (Rocha et al. 2007), as osteodistrofias de origem nutricional (Carciofi \& Oliveira 2007) ou as lesões traumáticas, fica comprometido, assim como diminuem as chances de se estabelecer medidas terapêuticas efetivas.

Considerando o exposto, esse trabalho foi desenvolvido com o intuito de se estabelecer parâmetros para o diagnóstico radiológico do quati, a partir da descrição comparativa da sua morfologia radiográfica e da disponibilização de imagens das suas principais projeções radiográficas.

\section{MATERIAL E MÉTODOS}

Foram utilizados 13 quatis (Nasua nasua) adultos, oriundos do Parque Zoológico da Universidade Federal do Mato Grosso (UFMT), campus Cuiabá/MT. Os animais foram submetidos a um jejum sólido de 12 horas e hídrico de 2 horas, anestesiados com a associação de tiletamina e zolazepan na dose de $7 \mathrm{mg} /$ $\mathrm{kg}$, pela via intra-muscular. 0 projeto foi aprovado pelo Comitê de Ética em Pesquisa Animal (CEPA)-UFMT sob protocolo n을 $3108.020341 / 11-0(\mathrm{~A})$.

Para a realização das radiografias foi utilizado aparelho de $\mathrm{Rx}$ fixo com $300 \mathrm{~mA} / 120 \mathrm{Kv}$, écrans e filmes de média velocidade e revelação manual nas dependências do Hospital Veterinário da UFMT. A cabeça, pescoço, tórax, abdome e pelve foram radiografados sob as projeções ventrodorsal e laterolateral e os membros torácicos e pélvicos sob as projeções mediolateral e anteroposterior. Adicionalmente, foram realizadas projeções oblíquas das mandíbulas e dos maxilares.

Um indivíduo idoso da espécie, morto por causas naturais, foi submetido a processo de maceração para posterior montagem do esqueleto. A pele e a musculatura foram retiradas através de desossa. Em seguida, o esqueleto foi submetido à técnica de maceração em água corrente para retirada mais ampla dos tecidos moles. Os ossos foram clareados com água oxigenada (10 volumes) e as partes que possuíam tecidos fibrosos ou cartilaginosos foram submetidas, logo após o descarne mais refinado, à técnica de Giacomini, sem passar pela técnica de maceração.

A análise das radiografias foi conduzida a partir da comparação das estruturas dos animais estudados com peça anatômica de um quati e com a anatomia conhecida de caninos, de acordo com nomenclatura utilizada por Dyce et al. (1997). Foram consideradas nessa avaliação a morfologia e a localização das estruturas anatômicas.

\section{RESULTADOS}

O seio frontal dos quatis apareceu bastante desenvolvido, se estendeu desde o osso frontal até as adjacências do osso incisivo. No seu aspecto mais cranial foi observado com formato triangular agudo entre os ossos nasais e os maxilares (Fig.1A). Apresentou vários septos ósseos bem definidos, o que lhe conferiu aspecto radiográfico reticulado (Fig.1D). Os machos adultos apresentaram crista sagital externa bastante proeminente, com cerca de $0,5 \mathrm{~cm}$ de altura, desde o osso occipital até o $1 / 3$ caudal do osso frontal. A dentição observada foi I 3/3, C1/1, P4/4, M2/2 = 40 (Fig.1B-D), com dentes molares uniformes, bem desenvolvidos e adaptados para mastigação e os caninos longos e proeminentes (Fig. $1 \mathrm{~A}, \mathrm{C})$.

Essa espécie apresentou sete vértebras cervicais, quinze torácicas (Fig.2A e 3A), cinco vértebras lombares e três sacrais (fusionadas) (Fig.2B e 3B). A quantidade de pares de costelas variou entre os animais. A maioria dos animais (9) apresentou 14 pares de costelas bem definidas nas radiografias (Fig. 3A). Os demais continham também um 15ํㅜ conjunto de costelas rudimentares.

Os ossos dos membros torácicos apareceram curtos, fortes, com o antebraço levemente arqueado. A escápula apresentou formato triangular e o úmero mostrou o epicôndilo medial muito proeminente (Fig.4A). Apresentaram cinco dígitos bem desenvolvidos, com o $1^{\text {o }}$ e o $5^{\text {o }}$ dígitos mais curtos que os demais. As falanges proximais mostraram cerca de $2 / 3$ do comprimento dos metacarpos, exceto

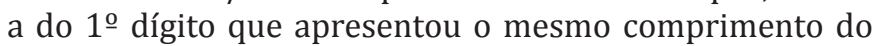
metacarpo correspondente. A falange média estava ausente no $1^{\text {o }}$ dígito e o comprimento das demais falanges correspondeu a aproximadamente metade da medida das falanges proximais (Fig.3B). No membro pélvico os ossos tiveram aparência similar aos da mão, entretanto os dígitos estavam mais próximos entre si, o que conferiu um aspecto mais alongado ao pé (Fig.5A,B).

O coração apresentou formato ovóide, aproximadamente $45^{\circ}$ de angulação entre o seu eixo longitudinal e o esterno e localização levemente voltada para a esquerda da linha média. Já a sua localização oscilou entre o 3 o e o 7ำ par de costelas, na projeção lateral (Fig.2A). 0 fígado localizou-se na parte interna da porção intratorácica do abdome, ocupou uma área triangular entre o diafragma, parede ventral do abdome e o estômago na projeção lateral (Fig.2B). A forma e a localização do estômago e do intestino grosso (Fig.2B e 3B) puderam ser determinadas pela presença de 

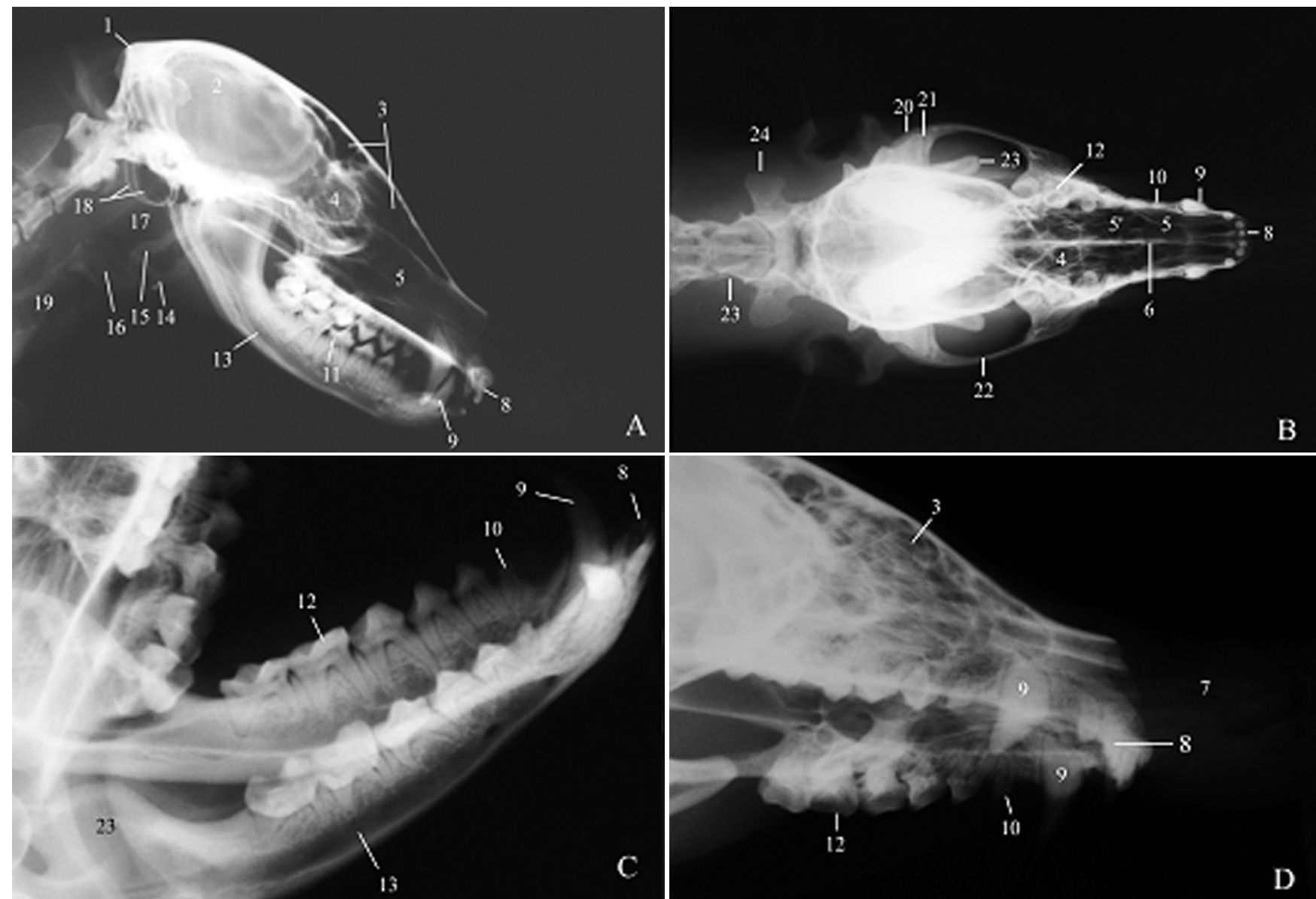

Fig.1. (A) Radiografia laterolateral do crânio e pescoço de quati fêmea. (B) Radiografia ventrodorsal, com boca aberta e Rx oblíquo de crânio de quati macho. (C) Radiografia oblíqua da mandíbula de quati fêmea. (D) Radiografia oblíqua dos maxilares de quati fêmea. (1) Osso occipital, (2) Encéfalo, (3) Seios frontais, (4) Conchas etmoidais, (5) Fossas nasais, (5') sobreposição de fossa nasal e seio frontal, (6) Septo nasal, (7) Focinho, (8) Dentes incisivos, (9) Dentes caninos, (10) 1o dente pré-molar, (11) 4o dente pré-molar, (12) $1^{\text {o }}$ dente molar, (13) Ramo horizontal da mandíbula, (14) Ossos hioides, (15) Palato mole, (16) Laringe, (17) Faringe, (18) Bolhas timpânicas, (19) Traquéia, (20) Osso temporal, (21) Articulação temporomandibular, (22) Arco zigomático, (23) Processo coronóide, (24) Atlas, (25) Axis.
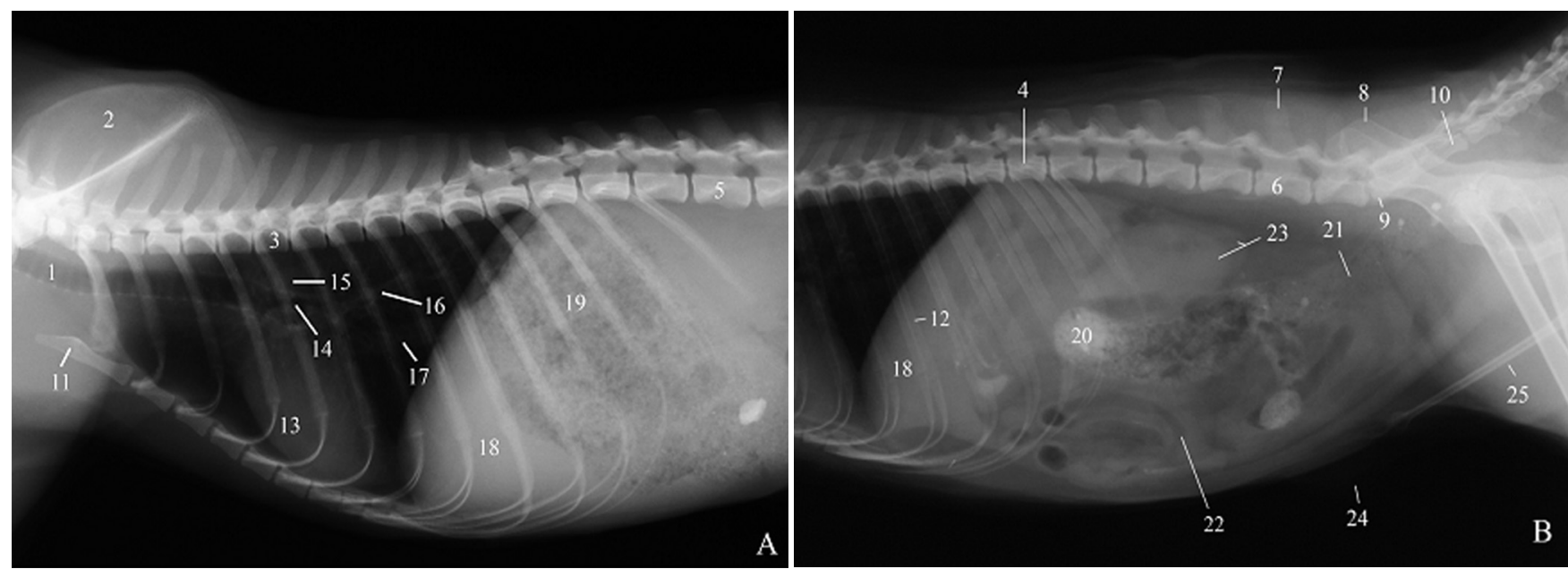

Fig.2. (A) Radiografia laterolateral do tórax de quati fêmea. (B) Radiografia laterolateral do abdome e pelve de quati macho. (1)Traquéia, (2) Escápula, (3) 6a vértebra torácica, (4) 14aa vertebra torácica, (5) 1a vértebra lombar, (6) 4a vértebra lombar, (7) Processo espi-

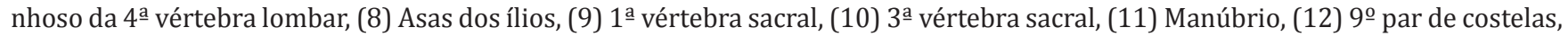
(13) Coração, (14) Bifurcação da traquéia, (15) Artéria aorta, (16) Vasos pulmonares dos lobos caudais, (17) Veia cava caudal, (18) Fígado, (19) Estômago, (20) Cólon transverso, (21) Cólon descendente, (22) Alça do intestino delgado, (23) Rins, (24) Prepúcio, (25) Osso peniano. 

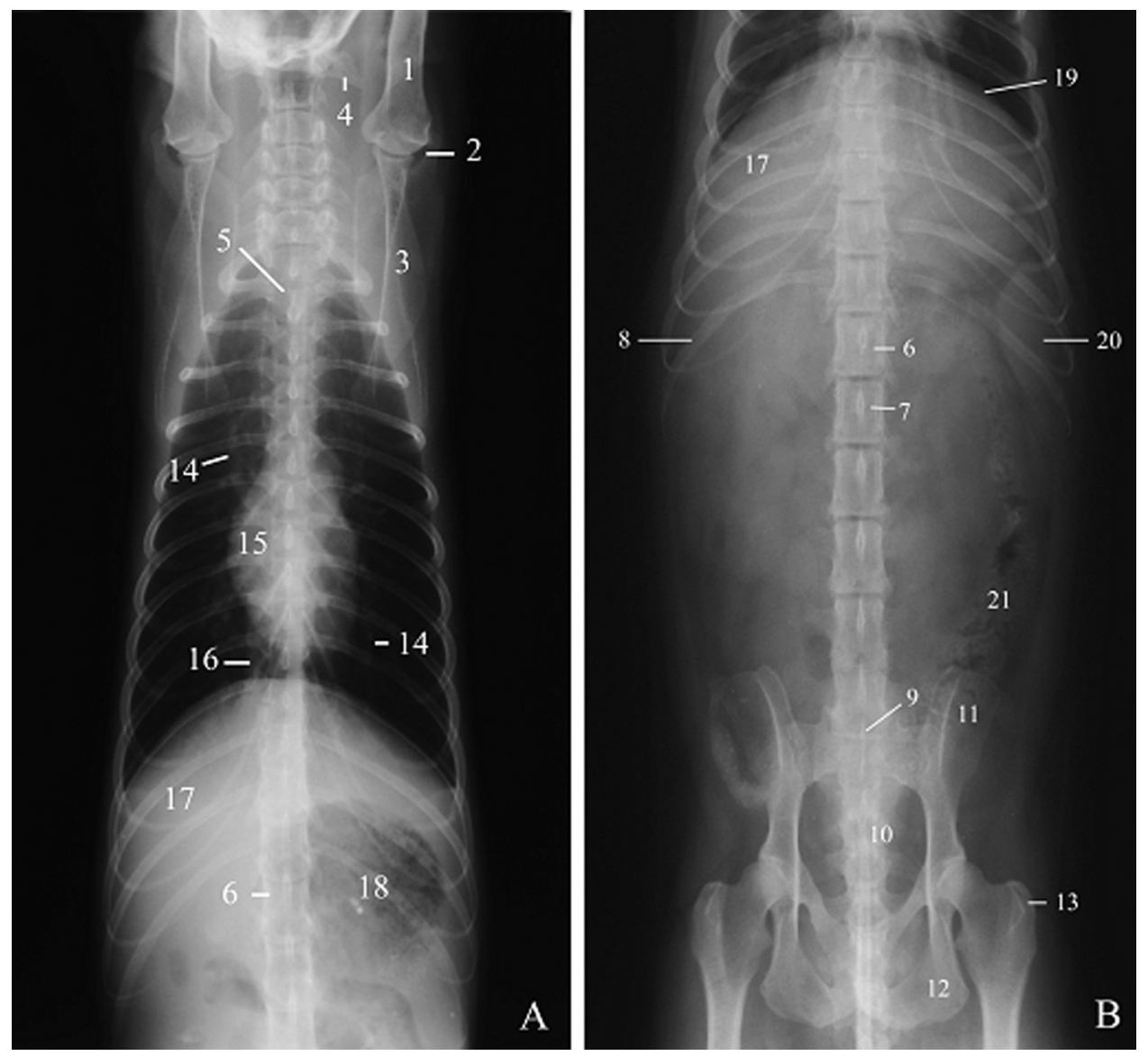

Fig.4. (A) Radiografia craniocaudal do membro anterior direito de quati macho. (B) Radiografia dorsopalmar de quati macho. (1) Escápula, (2) Articulação do ombro, (3) Úmero, (4) Fossa do olecrano (com forame supratroclear), (5.) Olecrano, (6) Epicôndilo medial, (7) Articulação do cotovelo, (8) Processo coronóide medial, (9) Ulna (proximal), (10) Rádio, (11) Acessório do carpo, (12) Ulnar do carpo, (13) Radial do carpo, (14) 5o metacarpiano, (15) Falange proximal, (16) Falange média, (17) Falange distal, (18) Ossos sesamóides proximais.
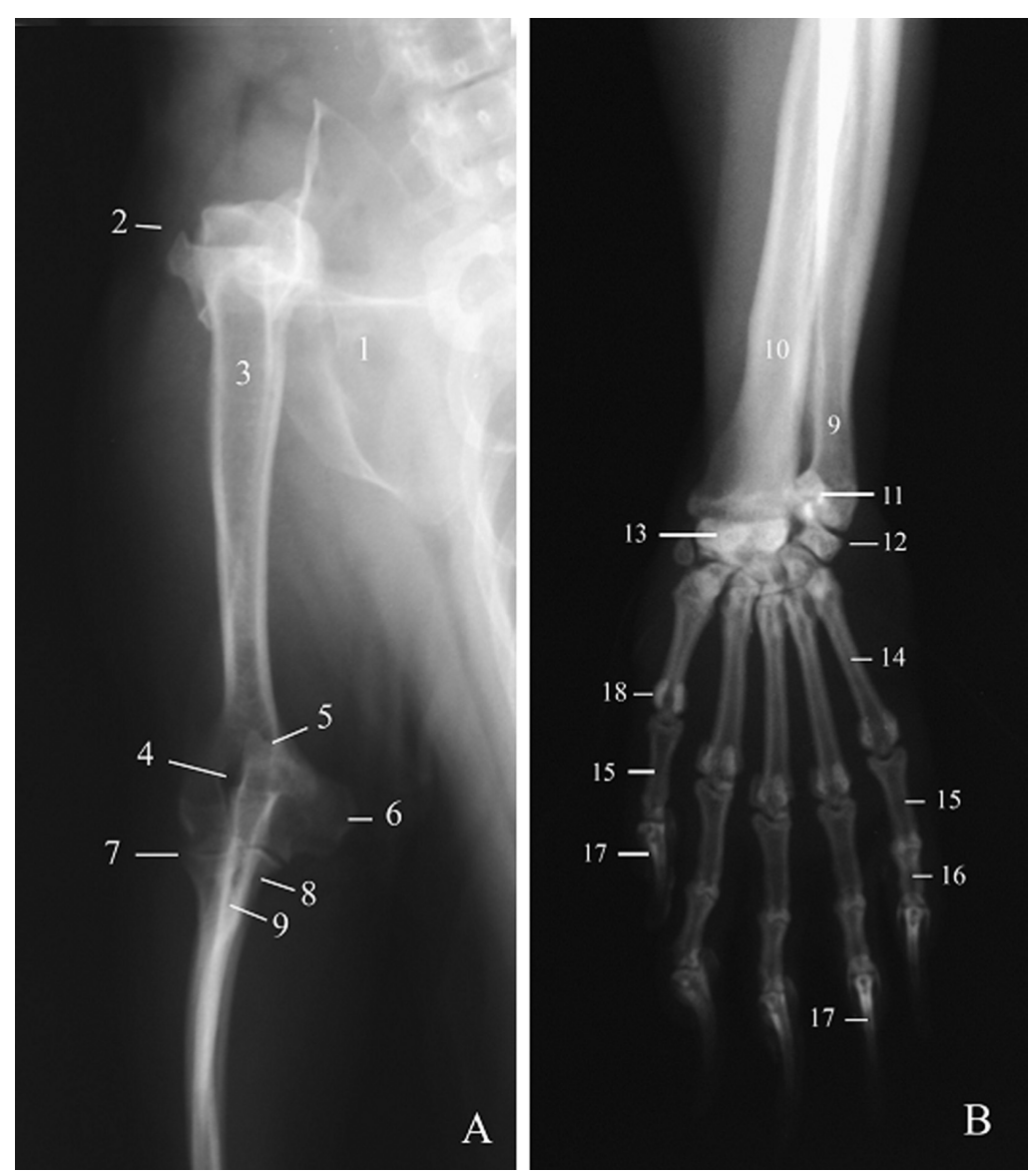

Fig.3. (A) Radiografia ventrodorsal de pescoço e tórax de quati fêmea. (B) Radiografia ventrodorsal de abdome e pelve de quati fêmea. (1) Úmero, (2) Articulação do ombro, (3) Escápula, (4) Atlas, (5) 1avértebra torácica, (6) 15a vértebra toráci-

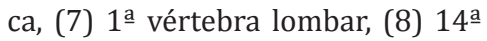
costela direita, (9) 1aㅡ vértebra sacral, (1). 1avértebra coccígea, (11) Asa do ílio esquerdo, (12) Ísquio esquerdo, (13) Trocanter maior do fêmur esquerdo, (14) Vasos pulmonares, (15) Coração, (16) Veia cava caudal, (17) Fígado, (18) Estômago, (19) Silhueta diafragmática, (20) Baço, (21) Cólon descendente. 


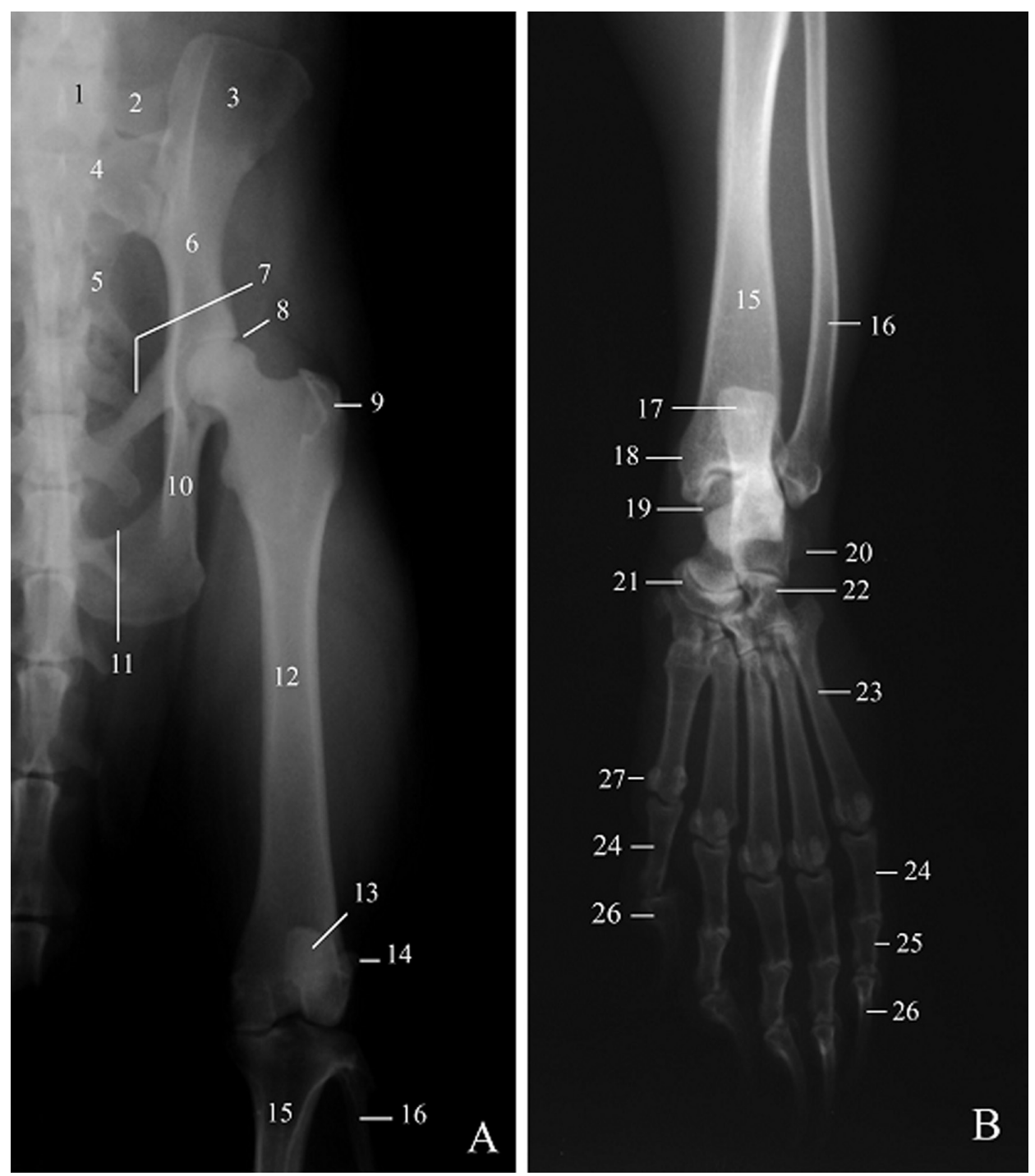

Fig.5. (A) Radiografia ventrodorsal da pelve e fêmur esquerdo de quati macho. (B) Radiografia dorsoplantar do membro posterior esquerdo de quati fêmea. (1) 6 vértebra lombar, (2) processo transverso da 6⿳a vértebra lombar, (3) Asa do ílio, (4) 1a vértebra sacral, (5) 3a vértebra sacral, (6) Corpo do ílio, (7) Púbis, (8) Articulação coxofemoral, (9) Trocanter maior, (10) Ísquio, (11) Forame obturado, (12) Fêmur, (13) Patela, (14) Sesamóideo lateral, (15) Tíbia, (16) Fíbula, (17) Tuberosidade do calcâneo, (18) Maléolo medial, (19) Tálus, (20) Calcâneo, (21) Central do tarso, (22) Quarto társico, (23) 5o metatarsiano, (24) Falange proximal, (25) Falange média, (26) Falange distal, (27) Ossos sesamóides proximais.

conteúdo intraluminal, sólido ou gasoso. 0 estômago se localizou dorsocaudalmente ao fígado e acompanhou o sentido das últimas costelas nas projeções laterais (Fig.2A) e nas radiografias ventrodorsais a "bolha estomacal" marcou com frequência a região fúndica (Fig.3A). 0 baço apareceu com formato predominantemente triangular caudolateralmente ao estômago na projeção ventrodorsal (Fig.3B), e caudal ao fígado nas radiografias laterais. Os rins não foram identificados em um percentual considerável dos animais examinados. Dos 13 animais avaliados eles puderam ser verificados em apenas seis, principalmente nas projeções laterais. A aparência deles lembrou o formato do feijão, localizaram-se com maior frequência entre a $2^{\underline{a}}$ e a $4^{\mathrm{a}}$ vértebras lombares e apareceram parcialmente sobrepostos na maioria das radiografias laterais (Fig.2B).

\section{DISCUSSÃO E CONCLUSÕES}

0 esqueleto do quati mostrou-se proporcionalmente mais longilíneo do que o dos caninos, ao se considerar a relação do esqueleto axial com o apendicular. Nessa espécie, o número de vértebras cervicais e sacrais observado foi idêntico ao descrito para caninos (Quadro 1), entretanto, esses animais apresentaram duas vértebras torácicas a mais e duas lombares a menos. Os números observados nesse trabalho foram similares aos de outros estudos conduzidos em quatis (Gregores 2006). Porém, o número de seis vértebras lombares, apontado como variação normal pelo autor foi observado em apenas um animal desse estudo.

Apesar de semelhante o crânio do quati diferiu em alguns aspectos do crânio do cão. Os seios frontais mostraram-se mais amplos, com extensão cranial entre o osso 
Quadro 1. Quantidade de vértebras, costelas, esternébras e dentes de caninos e de quatis

\begin{tabular}{lcc}
\hline Estruturas anatômicas & \multicolumn{2}{c}{ Quantidade } \\
\cline { 2 - 3 } & Quati & Cão* \\
\hline Vertebras cervicais & 7 & 7 \\
Vertebras torácicas & 15 & 13 \\
Vertebras lombares & $5-6$ & 7 \\
Vertebras sacrais & 3 & 3 \\
Costelas & $14-15$ & 13 \\
Dentes incisivos & $3 / 3$ & $3 / 3$ \\
Dentes caninos & $1 / 1$ & $1 / 1$ \\
Dentes pré-molares & $4 / 4$ & $4 / 4$ \\
Dentes molares & $2 / 2$ & $2 / 3$
\end{tabular}

* Dyce et al. (1996).

maxilar e o nasal, e numerosos septos bem evidentes. Entre os caninos essas cavidades apresentam geralmente apenas três compartimentos, sua maior extensão é limitada ao osso frontal (Dyce et al. 2010) e os septos são discretos nas radiografias laterolaterais e rostrocaudais (Kealy et al. 2012). A dentição observada foi similar à descrita por Pieri et al. (2011) para quatis, porém, mostrou-se distinta da estabelecida para caninos, principalmente com relação aos dentes molares e pré-molares. Além da diferença numérica verificada entre os dentes molares (Quadro 1), ficou evidente a uniformidade de tamanho entre os molares e o $3^{\circ}$ e $4^{\circ}$ pré-molares, tanto na arcada superior quanto na inferior. Esses dentes apresentaram coroa dentária relativamente plana e desenvolvida, bem adaptados para a mastigação, conforme descreveu Teixeira \& Ambrosio (2007), compatível com sua condição de onívoro e predileção alimentar por animais invertebrados e frutas (Gompper \& Decker 1998).

As principais diferenças entre a morfologia radiográfica dos membros de quatis e de caninos se limitaram às mãos e aos pés. Os quatis apresentaram 5 dígitos bem desenvolvidos e os metacarpos e os metatarsos levemente mais curtos do que os dígitos correspondentes. Já os caninos apresentam metacarpos e metatarsos proporcionalmente mais longos do que os dígitos e o $1^{\text {o }}$ dígito da mão é proporcionalmente menor do que os dos quatis. Porém esse dedo, assim como no quati, também apresenta apenas duas falanges, conforme descrito por Ellenport (1986). Por outro lado, o 1o dígito do pé do canino muitas vezes está ausente e quando presente varia de tamanho e tem uma ou duas falanges. Além disso, um sexto dedo livre pode ser observado nas raças grandes, aderido ao tecido fibroso (Ellenport 1986). Tais diferenças têm provavelmente relação direta com a forma de ambulação das espécies. Os quatis são plantígrados (Teixeira \& Ambrosio 2007), apoiam completamente as mãos e os pés durante a ambulação, o que lhes permite escalarem árvores e obstáculos íngremes. Tanto que em algumas regiões estudadas os animais são predominantemente arborícolas (Beisiegel 2001). Já os caninos são digitígrados e caminham diretamente sobre os dígitos. A ulna e fíbula mostraram-se proporcionalmente mais robustas, os ossos do púbis mais angulados e a asa do ílio mais larga e proeminente do que a dos caninos. Os ossos radial do carpo, ulnar do carpo, acessório do carpo calcâneo, tálus, quarto osso társico e central do tarso apresentaram formato e relações anatômicas similares aos descritos por Dyce et al. (2010) para caninos.
0 osso peniano do quati diferiu do cão tanto no comprimento quanto no formato. No quati o osso mostrou-se proporcionalmente mais longo, apresentou diâmetro regular em toda a sua extensão e uma dilatação na extremidade distal, similar ao descrito por Franciolli et al. (2007). No cão, o osso é sulcado ventralmente, afila-se em direção à sua extremidade distal e não atinge o ápice peniano (Dyce et al. 2010).

Apesar de não ter sido conduzida a dissecação de tecidos moles nesse estudo, pôde-se verificar que a localização e os aspectos anatômico e radiográfico das principais vísceras torácicas e abdominais foram similares às descritas para caninos por Dyce et al. (2010) e Kealy et al. (2012). A localização cardíaca, apontada nesse estudo entre 30 e o 7으 par de costelas foi similar à descrita por Turbino (2010) em estudo sobre a mensuração cardíaca pelo método VHS (vertebral heart size) em quatis.

Agradecimentos.- À FAPEMAT pela concessão de bolsa modalidade Residência Médica Veterinária e aos funcionários do Parque Zoológico da Universidade Federal de Mato Grosso pelo suporte.

\section{REFERÊNCIAS}

Beisiegel B.M. 2001. Notes on the coati, Nasua nasua (Carnívora: Procyonidae) in a Atlantic Forest area. Braz. J. Biol. 61(4):689-692.

Carciofi A.C. \& Oliveira L.D. 2007. Doenças nutricionais, p.834-865. In: Cubas S.Z., Silva J.C.R. \& Catão-Daias J.L. (Eds), Tratado de Animais Selvagens: medicina veterinária. Editora Roca, São Paulo. 1376p.

Cubas S.Z., Silva J.C.R. \& Catão-Dias J.L. 2007. Tratado de Animais Selvagens: medicina veterinária. Editora Roca, São Paulo.

Dyce K.M., Sack W.O. \& Wensing C.J.G. 2010. Tratado de Anatomia Veterinária. $4^{a}$ ed. Elsevier, Rio de Janeiro. 872p.

Ellenport C.R. 1986. Ostologia do carnívoro, p.1337-1412. In: Getty R. (Ed.), Sisson e Grossman Anatomia dos Animais Domésticos. 5a ed. Guanabara Koogan, Rio de Janeiro.

Fonseca Pinto A.C.B.C. 2007. Radiologia, p.896-919. In: Cubas S.Z., Silva J.C.R. \& Catão-Dias J.L. (Eds), Tratado de Animais Selvagens: medicina veterinária. Editora Roca, São Paulo.

Franciolli A.L.R, Costa G.M., Mançanares C.A.F., Martins D.S., Ambrósio C.E., Miglino M.A. \& Carvalho A.F. 2007. Morfologia dos órgãos genitais masculinos de quati (Nasua nasua Linnaeus, 1766). Biotemas 20(1):27-36.

Freitas E.P., Rahal S.C., Teixeira C.R., Teixeira R.H., Mendes G.M. \& Gioso M.A. 2008. Oral cavity evaluation and dental chart registration of coati (Nasua nasua) in captivity. J. Vet. Dent. 25(2):110-7.

Gompper M.E. \& Decker D.M. 1998. Nasua nasua. Mammalian Species 580:1-9.

Gregores G.B. 2006. Topografia vértebro-medular e anestesia espinhal em quati (Nasua nasua). Dissertação de Mestrado, Universidade de São Paulo, São Paulo, SP. 70p.

Gregores G.B., Branco E., Carvalho A.F., Sarmento C.A.P., Oliveira P.C., Ferreira G.J., Cabral R., Fioretto E.T., Miglino M.A. \& Cortopassi S.R.G. 2010. Topografia do cone medular do quati (Nasua nasua Linnaeus, 1766). Biotemas 23(2):173-176.

Kealy J.K., McAllister H. \& Graham J.P. 2012. Radiologia e Ultra-sonografia do Cão e do Gato. 5ª ed. Elsevier, Rio de Janeiro, 580p.

Pieri N.C.G., Mançanares C.A.F., Bertassoli B., Lima J.M.N., Thomaz J.M. \& Carvalho A.F. 2011. Classificação morfofuncional dos dentes de quati, Nasua nasua. Pesq. Vet. Bras. 31(5):447-451.

Schebitz H. \& Wilkens H. 2000. Atlas de Anatomia Radiográfica do Cão e do Gato. 5a ed. Manole, Barueri. 244p.

Teixeira R.H.F. \& Ambrosio S. 2007. Procyonidae, p.571-583. In: Cubas S.Z., Silva J.C.R. \& Catão Dias J.L. (Eds), Tratado de Animais Selvagens: medicina veterinária. Editora Roca, São Paulo. 
Turbino N.C.M.R. 2010. Análise do traçado eletrocardiográfico e avaliação radiográfica da silhueta cardíaca, pelo método VHS (Vertebral Heart Size), de quatis (Nasua nasua Linnaeus, 1766) jovens e adultos mantidos em cativeiro e contidos quimicamente com tiletamina e zolazepam. Dissertação de Mestrado em Sanidade de Animais domésticos e Selvagens, Faculdade de Agronomia e Medicina Veterinária, Universidade Federal de Mato Grosso, Cuiabá, MT. 52p.

Kommers G.D., Ilha M.R.S. \& Barros C.S.L. 1999. Dioctofimose em cães: 16 casos. Ciência Rural 29(3):517-522.

Rocha V.C.M., Corrêa S.H.R., Oliveira E.M.D., Rosales Rodriguez C.A., Ramos M.C.C., Fedullo J.D.L., Teixeira R.H.F., Catão Dias J.L., Fiorio W.A.B., Matro- ne M., Moraes Z.M., Souza G.O., Ikuta C.Y., Vejarano M.P. \& Ferreira Neto J.S. 2007. Isolamento e identificação de Mycobacterium kansasii a partir de lavado traqueal de quati (Nasua nasua) mantido em cativeiro no Brasil. Biológico, São Paulo, 69(2):113-198.

Milanelo L., Moreira M.B., Fitorra L.S., Petri B.S.S., Alves M. \& Santos A.C. 2009. Occurrence of parasitism by Dioctophyma renale in ring-tailed coatis (Nasua nasua) of the Tiete Ecological Park, São Paulo, Brazil. Pesq. Vet. Bras. 29(12):959-962.

Santos A.C., Bertassoli B.M., Oliveira V.C., Carvalho A.F., Rosa R.A. \& Mançanares C.A.F. 2010. Morfologia dos músculos do ombro, braço e antebraço do quati (Nasua nasua Linnaeus, 1958). Biotemas 23(3):167-173. 